Gefässchirurgie 2020 · 25:417-422 https://doi.org/10.1007/s00772-020-00686-5 Angenommen: 16. August 2020 Online publiziert: 2 . September 2020 (c) Der/die Autor(en) 2020

\section{Einführung}

Bereits im Dezember 2019 wurde über eine in der Millionenmetropole Wuhan in China ausgebrochene Epidemie berichtet, die durch schwere Pneumonien und damit assoziierte Todesfälle charakterisiert und durch einen neuen Coronavirus (Severe Acute Respiratory Syndrome - Corona Virus 2, SARS-CoV-2) ausgelöst waren. Die nachfolgende weltweite Ausbreitung im Sinne einer Pandemie der dann Corona-Virus-Disease19 (COVID-19) genannten Infektion erfolgte u. a. mit einem ersten europäischen Hotspot in Norditalien im Februar 2020 [2]. Aufgrund der geografischen Nähe Österreichs zu Norditalien mit häufiger medialer Berichterstattung über die äußerst problematische Situation im Nachbarland waren Behörden und Bevölkerung bereits erheblich alarmiert, als am 25. Februar 2020 erstmals in Österreich zwei Personen (italienischstämmige Hotelmitarbeiter in Innsbruck, beide kamen aus der Provinz Bergamo) positiv auf COVID-19 getestet wurden. Nachfolgend stieg die Anzahl der positiv auf COVID-19 getesteten Personen in Österreich rasch an, v. a. im Bundesland Tirol, sodass am 11. März 2020 die österreichische Bundesregierung die Schließung aller nicht systemnotwendigen Einrichtungen inkl. der Schulen und Universitä-

J. Klocker ${ }^{1} \cdot$ A. Frech ${ }^{1} \cdot$ A. Gratl ${ }^{1} \cdot$ M. Thaler ${ }^{2} \cdot$ I. Khosravi' ${ }^{2}$ M. Liebensteiner ${ }^{2}$. M. Kluckner ${ }^{1}$ W. Hofmann ${ }^{3} \cdot$ A. Assadian ${ }^{4}$ Ö̈sterreichische Gesellschaft für Gefäßchirurgie (ÖGG)

${ }^{1}$ Universitätsklinik für Gefäßchirurgie, Medizinische Universität Innsbruck, Innsbruck, Österreich

${ }^{2}$ Universitätsklinik für Orthopädie, Medizinische Universität Innsbruck, Innsbruck, Österreich

${ }^{3}$ Abteilung für Gefäßchirurgie, LKH Feldkirch, Feldkirch, Österreich

${ }^{4}$ Abteilung für Gefäßchirurgie, Wilhelminenspital Wien, Wien, Österreich

\title{
Operieren, Absagen, Verschieben oder Selektionieren?
}

\section{Ergebnisse einer Umfrage unter gefäßchirurgischen Zentren während der Covid-19-Krise in Österreich}

ten beschloss. Am 12. März war dann der erste COVID-19-Todesfall in Österreich zu verzeichnen und ab 15. März 2020 wurde dann eine österreichweite generelle Ausgangsbeschränkung („Lockdown“) umgesetzt.

$\mathrm{Zu}$ diesem Zeitpunkt war allerdings unklar, wie viele der positiv auf COVID-19 getesteten Personen tatsächlich Krankheitssymptome entwickeln würden, wie viele davon in einem Krankenhaus behandelt und v.a. wie viele künstlich beatmet werden müssen. Es wurde anhand der damals vorliegenden Daten [4] davon ausgegangen, dass bis zu $14 \%$ der positiv getesteten Patienten hospitalisiert werden müssen und bis $\mathrm{zu} 6 \%$ einen Beatmungsplatz benötigen. Es war daher auch in Österreich schnell nötig, Ressourcen für Patienten mit schwerer COVID-19-Infektion freizumachen, indem nicht dringliche operative oder interventionelle Eingriffe vorläufig ausgesetzt (abgesagt oder verschoben) wurden. Insgesamt war die Einsparung limitierter Ressourcen hierbei aber nicht nur auf Bettenkapazitäten auf Normal- und Intensivstationen bezogen, sondern auch auf einzelne medizinische Verbrauchsgüter (z.B. OPund FFP-Masken, Handschuhe, Desinfektionsmittel und Schutzkittel). Das „Fernhalten“ von Patienten mit nicht dringlichen bzw. nicht akuten Erkran- kungen von Krankenhäusern diente zudem natürlich auch der Vermeidung einer potenziellen Exposition (COVID19-positive Patienten bzw. Mitarbeiter).

Wie die Reduktion operativer oder interventioneller gefäßchirurgischer Eingriffe während der Phase des aufgrund der COVID-19-Pandemie ab 15. März 2020 angeordneten Lockdowns in Österreich erfolgte, welche gefäßchirurgischen Operationen weiter durchgeführt, abgesagt oder verschoben wurden, und wie einheitlich oder uneinheitlich dieses Vorgehen in verschiedenen gefäßchirurgischen Zentren des Landes war, wurde mittels eines Onlinefragebogens erfasst. Einzelne Aspekte des Fragebogens und der Ergebnisse werden im Folgenden dargestellt.

In Österreich gab es - zum Zeitpunkt unserer Umfrage - keine Stellungnahme der eigenen Fachgesellschaft (ÖGG), und auch die Österreichische Gesellschaft für Chirurgie hat für die gefäßchirurgischen Indikationen keine Empfehlungen zur Vorgehensweise in der Phase der COVID-19-Pandemie veröffentlicht.

\section{Umfrage mittels Onlinefrage- bogen in Österreich}

Am 10. April 2020 wurden die Abteilungsleiter von insgesamt 15 österreichischen gefäßchirurgischen Zentren (Ab- 
teilungen mit ausschließlicher Ausrichtung auf Gefäßchirurgie oder chirurgische Abteilungen mit einem gefäßchirurgischen Schwerpunkt entsprechend dem Österreichischen Strukturplan Gesundheit/ÖSG 2017; alle in öffentlich-rechtlichen Zentral- oder Schwerpunktkrankenhäusern) per Mail mit erklärendem Begleitschreiben des Vorstands der Österreichischen Gesellschaft für Gefäßchirurgie (ÖGG) eingeladen, an einer kostenlosen anonymisierten Onlineumfrage über die aktuellen Implikationen der COVID-19-Pandemie auf die Patientenversorgung „,in den letzten Wochen“ teilzunehmen. Die Umfrage wurde für Aspekte des Fachgebiets Gefäßchirurgie adaptiert aus einer zeitgleichen Befragung im Fachgebiet Orthopädie $[5,7]$ und mittels SurveyMonkey (http://www.surveymonkey. com), einem kostenlos verfügbaren Onlineumfragetool, erstellt. Da die Umfrage keinerlei Einzeldaten von Patienten betraf, war kein Votum einer Ethikkommission verpflichtend.

Insgesamt haben 12 von 15 (80\%) befragten gefäßchirurgischen Zentren den Onlinefragebogen in den Tagen zwischen 10. April bis spätestens 20. April beantwortet, wobei für die Auswertung nicht ersichtlich war, welche Zentren geantwortet haben. Auch war nicht ersichtlich, ob die Beantwortung durch die Abteilungsleiter selbst oder Mitarbeiter erfolgte. Die Auswertung erfolgte also verblindet und anonymisiert (dies wurde mit den einzelnen Abteilungen vorab so vereinbart).

Die Fragen waren entweder als Multiple-Choice $(n=9)$, als Einzelantwort $(n=4)$ oder als offene Fragen mit möglicher Textantwort $(n=2)$ formuliert und kreisten um 3 Themenbereiche: Implikationen der COVID-19-Pandemie und der hierzu durch die österreichischen Behörden erlassenen Bestimmungen („nationwide shutdown“) auf (1) das medizinische Fachpersonal, (2) die ambulante und stationäre Patientenversorgung, (3) die operative und interventionelle Patientenversorgung.

In der nachfolgenden Darstellung werden vornehmlich Aspekte der operativen und interventionellen Patientenversorgung behandelt, konkret, ob Eingriffe weiterhin generell oder ledig- lich selektiv durchgeführt, verschoben oder überhaupt abgesetzt wurden. Wie sich (im Nachhinein) herausgestellt hat, waren die Begriffe „abgesetzt“ und „verschoben" schlecht zu differenzieren, wurden also vielfach nahezu synonym verwendet, da das „Absetzen“ einer OP (ein für alle Mal) selten anzunehmen ist und das „Verschieben“ allerdings in vielen Fällen mit unklarem und nicht festgelegtem Zeitfenster erfolgen musste, also de facto zunächst einem „Absetzen“ entsprach (zum Zeitpunkt der Befragung war die zeitliche Befristung der OP-Einschränkungen seitens der Behörden nicht absehbar).

\section{\) Die zeitliche Befristung der OP-Einschränkungen war nicht absehbar}

Entsprechend wurden auch im Onlinefragebogen die einzelnen Institutionen befragt, wie sie die Befristung der Einschränkungen der OP-Kapazitäten einschätzen. Hierbei wurden vordefinierte Zeitintervalle abgefragt: „5 bis 8 Wochen“, „9 bis 12 Wochen“, „3 bis 6 Monate“ oder „6 bis 9 Monate“.

Bereits mit Stichtag 22. April 2020, also bald nach der Schließung der Onlinebefragung, wurden vonseiten des Bundesministeriums für Soziales, Gesundheit, Pflege und Konsumentenschutz (BMSGPK) Empfehlungen zur schrittweisen Wiederaufnahme von aufgrund der COVID-19-Pandemie eingestellten bzw. reduzierten elektiven Tätigkeiten in Krankenanstalten herausgegeben, die dann aber in weiterer Folge erst schrittweise in den einzelnen Bundesländern Österreichs und auch mit regionalen Unterschieden umzusetzen waren.

\section{Ergebnisse der Umfrage - COVID-19-Fälle (Patienten und Mitarbeiter)}

Alle Krankenhäuser waren mit positiv auf COVID-19 getesteten Patienten konfrontiert, jedoch hatten nur $25 \%$ der gefäßchirurgischen Abteilungen positiv auf COVID-19 getestete Patienten zu behandeln. $75 \%$ der Krankenhäuser hatten positiv aufCOVID-19 getestete Mitarbeiter, allerdings nur $33 \%$ der gefäßchirurgischen Abteilungen.

\section{Ergebnisse der Umfrage - OPs und Interventionen während des landesweiten Shutdowns}

Alle Abteilungen berichteten, dass die Anzahl der während der COVID-19Pandemie operierten Patienten drastisch reduziert wurde. Elektive Eingriffe wurden in allen gefäßchirurgischen Abteilungen entweder überhaupt gestoppt (für ambulante/tagesklinische OPs in $58 \%$ der Zentren; für stationäre OPs in $42 \%$ der Zentren) oder selektiv eingeschränkt (für ambulante/tagesklinische OPs in $42 \%$ der Zentren; für stationäre OPs in $58 \%$ der Zentren).

Die Befristung der Einschränkungen der OP-Kapazitäten (vordefinierte Zeitintervalle) haben die einzelnen Abteilungen sehr unterschiedlich eingeschätzt: ,5 bis 8 Wochen" ( $17 \%)$, „9 bis 12 Wochen“ (33\%), „3 bis 6 Monate“ (17\%) oder „6 bis 9 Monate“ (33\%).

\section{》) Zum Zeitpunkt der Umfrage berichtete keine Institution über fehlende Kapazitäten}

Zum Zeitpunkt der Umfrage berichtete keine Institution über fehlende Kapazitäten bezüglich verfügbarer Betten (sowohl auf Normalstationen als auch auf Intermediate Care oder Intensivstationen) zur Behandlung von Patienten mit COVID19-Infektion. Allerdings wurden in der Hälfte der Institutionen die den gefäßchirurgischen Abteilungen zugeordneten Bettenressourcen reduziert. Als Folge der antizipierten limitierten Ressourcen in der Gefäßchirurgie wurden folgende Pathologien nahezu einheitlich in allen Zentren gestoppt oder verschoben: symptomfreie Karotisstenose (Endarteriektomie: in $92 \%$ der Zentren gestoppt oder verschoben; Karotisstent: in allen Zentren, die diese durchführen, gestoppt oder verschoben), symptomfreies Aortenaneurysma mit Maximaldurchmesser $5,5-7 \mathrm{~cm}$ (in $92 \%$ der Zentren gestoppt oder verschoben), symptomfreie periphere Aneurysmen (in $92 \%$ der Zentren gestoppt oder verschoben), periphere 
Gefässchirurgie 2020 $25: 417-422$ https://doi.org/10.1007/s00772-020-00686-5

(c) Der/die Autor(en) 2020

J. Klocker · A. Frech · A. Gratl · M. Thaler · I. Khosravi · M. Liebensteiner · M. Kluckner · W. Hofmann · A. Assadian · Österreichische Gesellschaft für Gefäßchirurgie (ÖGG)

\section{Operieren, Absagen, Verschieben oder Selektionieren? Ergebnisse einer Umfrage unter gefäßchirurgischen Zentren während der Covid-19-Krise in Österreich}

\section{Zusammenfassung}

Gefäßchirurgische Zentren in Österreich

$(n=15)$ wurden Mitte April 2020 mit

erklärendem Begleitschreiben des Vorstands

der Österreichischen Gesellschaft für

Gefäßchirurgie (ÖGG) eingeladen, an einer

Onlineumfrage über die Implikationen der

COVID-19-Pandemie teilzunehmen. Insgesamt

12 Zentren ( $80 \%$ ) haben den Fragebogen ausgefüllt.

Alle Zentren waren mit positiv getesteten $\mathrm{Pa}$ tienten und $75 \%$ auch mit positiv getesteten Mitarbeitern konfrontiert. Deutlich seltener war dies jedoch an den gefäßchirurgischen Abteilungen (positiv getestete Patienten in $25 \% /$ Mitarbeiter in $33 \%$ ). Elektive Eingriffe wurden an allen Abteilungen entweder gestoppt (abgesagt oder verschoben) oder selektiv eingeschränkt. Dies betraf u. a. auch symptomfreie Patienten mit Karotisstenose, Aortenaneurysma kleiner $7 \mathrm{~cm}$, peripherem Aneurysma, PAVK im Stadium II nach Fontaine oder Varikose. In allen Zentren wurden weiterhin uneingeschränkt gefäßchirurgische Notfälle behandelt. Uneinheitlich war das Vorgehen bei chronischen Ulzera, chronischer Mesenterialinsuffizienz, symptomfreiem Aortenaneurysma größer $7 \mathrm{~cm}$ und in der Shuntchirurgie.

Insgesamt war v.a. die zum Zeitpunkt der OPVerschiebungen unklare Befristung der Maßnahmen problematisch, somit war auch das Risiko der abgesagten bzw. hinausgezögerten Behandlung nicht leicht abschätzbar. Für die Indikationen mit uneinheitlichem Vorgehen sollte unizentrisch und multizentrisch das Ergebnis der Patienten analysiert werden, auch hinsichtlich der mit der Verschiebung assoziierten psychologischen Belastung. Zudem bedürfen auch die aufgrund der COVID-19-Pandemie erfolgten Veränderungen im Alltag einer kritischen Auswertung, auch hinsichtlich demografischer und geografischer Unterschiede. Es ist anzunehmen, dass die Auswirkungen der COVID-19-Pandemie langfristig bedeutsam sind.

Schlüsselwörter COVID-19 · Gefäßchirurgie · OP-Verschiebung · OP-Triage · Umfrage

\section{Operate, cancel, postpone or select? Results of a survey of vascular surgery centers during the COVID- 19 pandemic in Austria}

\section{Abstract}

Regional centers performing vascular surgery in Austria $(n=15)$ were invited in mid-April 2020 by the Austrian Society of Vascular Surgery (ÖGG) to participate in a nationwide survey about implications of the COVID-19 pandemic. Ultimately, a total of 12 centers $(80 \%)$ answered the questionnaire. All centers were confronted with patients who tested positive for COVID-19 and 75\% also had medical personnel who were positive. In contrast, only $25 \%$ of the departments of vascular surgery had positively tested patients and $33 \%$ had positive staff members. In all departments of vascular surgery elective vascular procedures were either stopped (cancelled or deferred) or selectively limited, including patients with asymptomatic carotid stenosis, aortic aneurysms smaller than $7 \mathrm{~cm}$, peripheral arterial aneurysm, peripheral artery occlusive disease Fontaine stage II and varicosities. All centers continued to carry out operations for all types of vascular surgical emergencies. The strategies of the centers were heterogeneous for patients with chronic ulcers, chronic mesenteric insufficiency, asymptomatic aortic aneurysms larger than $7 \mathrm{~cm}$ and shunt surgery.

Decisions on surgery cancellation seemed to be particularly problematic due to the uncertain time period of the COVID-19 measures. As a consequence, the risk associated with cancellation or delayed treatment was difficult to assess. At present, especially indications with nonuniform management strategies need selective attention and additional analysis in single center and multicenter studies. In addition, patients might suffer from relevant psychological problems because of surgery cancellations. Changes in the daily routine due to the COVID-19 pandemic may have a long-term impact on health status and may show significant demographic and geographic variations.

\section{Keywords}

Severe acute respiratory syndrome coronavirus 2 - Vascular surgical procedures - Surgery cancellation $\cdot$ Surgery triage $\cdot$ Survey arterielle Verschlusskrankheit im Stadium II nach Fontaine (in allen Zentren gestoppt oder verschoben), Varikose (in allen Zentren gestoppt oder verschoben).

Im Gegensatz dazu wurden in allen gefäßchirurgischen Zentren weiterhin uneingeschränkt Patienten operiert, die eine akut behandlungsbedürftige arterielle Pathologie hatten: symptomatische Karotisstenose, akute Extremitätenischämie, symptomatisches oder rupturiertes
Aneurysma (aortoiliakal oder peripher), akutes Aortensyndrom, traumatische Aortenruptur, akute Mesenterialischämie bzw. traumatische oder iatrogene Gefäßverletzung. Des Weiteren wurden in $92 \%$ der Zentren in üblicher Weise weiterhin Patienten mit peripherer arterieller Verschlusskrankheit im Stadium III oder IV nach Fontaine behandelt, und auch weiterhin Major- und Minoramputationen durchgeführt. Auch wurden weiterhin in allen Institutionen zentralvenöse Katheter zur Hämodialyse und Portsysteme zur Chemotherapie angelegt.

Uneinheitlich war hingegen das Vorgehen der gefäßchirurgischen Zentren bei Patienten mit chronischen Ulzera (nur in $25 \%$ weiterhin behandelt, in $75 \%$ hingegen gestoppt bzw. verschoben), chronischer Mesenterialinsuffizienz (in 33\% weiterhin durchgeführt, in $67 \%$ hinge- 
gen gestoppt bzw. verschoben) oder symptomfreiem Aortenaneurysma mit Maximaldurchmesser größer als $7 \mathrm{~cm}$ (in $75 \%$ wurde eine offene oder endovaskuläre Behandlung weiterhin durchgeführt, in $25 \%$ verschoben). Auch in der Shuntchirurgie war das Vorgehen sehr unterschiedlich: $50 \%$ führten diese weiterhin durch, in den anderen $50 \%$ der Zentren wurde diese jedoch gestoppt oder verschoben.

\section{Erkenntnisse der Umfrage}

Die in Österreich in gefäßchirurgischen Zentren durchgeführte Onlineumfrage zeigt, dass in allen Institutionen Patienten mit COVID-19-Infektion behandelt wurden, aber vergleichsweise seltener $\mathrm{Pa}$ tienten und Mitarbeiter der gefäßchirurgischen Abteilungen betroffen waren. Das ist insbesondere auch deshalb bemerkenswert, da Patienten mit kardiovaskulären Erkrankungen, mit Diabetes oder mit arterieller Hypertonie als Hochrisikopatienten für eine fatal verlaufende COVID-19-Infektion gelten [6]. Sowohl Patienten als auch Gesundheitspersonal waren während der COVID-19Pandemie einem vergleichsweise hohen und potenziell lebensbedrohlichen Risiko ausgesetzt, dies auch aufgrund einer mangelhaften Vorbereitung der Institutionen.

\section{》) Die Institutionen waren auf die Pandemie nur unzureichend vorbereitet}

Die COVID-19-Pandemie und der deshalb erfolgte Lockdown stellte auch in Österreich das Gesundheitspersonal und die Patienten vieler Krankenhausabteilungen, insbesondere auch jene der chirurgischen Fachgebiete, vor vielfältige Herausforderungen: u.a. mussten elektive Indikationen zur operativen und interventionellen Behandlung und deren Dringlichkeit eingehend überprüft und hinterfragt werden. Hierzu waren zum Zeitpunkt der Entscheidungsfindung für die Fachexperten aber keine Erfahrungsberichte vorliegend („welche Operationen wie lange verschieben?"), das Zeitfenster für Verschiebungen war völlig unklar (einige Monate? ein halbes Jahr? noch länger?) und das Risiko für den Patienten aufgrund einer abgesagten bzw. hinausgezögerten Behandlung in vielen Fällen nicht leicht abschätzbar.

Entsprechend haben auch in der Beantwortung des Onlinefragebogens die einzelnen Institutionen die unklare Befristung der Einschränkungen der OPKapazitäten sehr unterschiedlich eingeschätzt. Konkret nahmen $50 \%$ der Institutionen an, dass diese zumindest 3 Monate oder länger andauern würde. Zum Zeitpunkt der Entscheidung zur OP-Verschiebung wurde die Befristung also (mit heutigem Wissen ex post) länger eingeschätzt, als sie tatsächlich war, da angenommen werden kann, dass viele Zentren ab Mai 2020 wieder zu einer „normalen Performance“ zurückkehren konnten.

Interessant scheint in diesem Zusammenhang die Publikation eines auf einer Expertenumfrage basierten Modells, das für die aktuelle COVID-19-Pandemie mittels Bayes'scher linearer Regression abschätzte, wie viele Fallabsagen elektiver Operationen weltweit innerhalb von 12 Wochen ,angenommenem Stillstand“ (für Elektivoperationen) zu erwarten wären: über 28 Mio. Operationen bzw. $72,3 \%$ aller Operationen wären betroffen. Selbst wenn - nach der COVID19-Pandemie - die OP-Kapazitäten um $20 \%$ gesteigert werden, würde die Aufarbeitung der verschobenen Operationen median 45 Wochen in Anspruch nehmen. Aus gefäßchirurgischer Sicht ist an dieser Publikation der COVIDSurg Collaborative zu kritisieren, dass (1) Europa und Zentralasien gemeinsam ausgewertet wurden (trotz anzunehmender beträchtlicher regionaler Unterschiede), und (2) die Gefäßchirurgie in der Kategorie "other specialties" gemeinsam mit der Herzchirurgie, Thoraxchirurgie, Neurochirurgie und Mammachirurgie (!) dargestellt wurde [3].

Unsere Onlineumfrage zeigt aber auch, dass die Entscheidung zum Verschieben bzw. Absetzen von gefäßchirurgischen Operationen und Interventionen in den unterschiedlichen Institutionen in vielen Fällen einheitlich erfolgte, gewissermaßen „nach einem logischen Konzept“: Notfälle und dringliche Fälle wurden weiterhin ohne Verzögerung behandelt, elektive Eingriffe regelhaft postponiert. Allerdings war die Entscheidung für einzelne Krankheitsbilder bzw. Indikationen uneinheitlich, u. a. die Shuntchirurgie (Shuntanlage bei funktionierendem HD-Katheter?), das Management von Patienten mit chronischen Ulzera (Revaskularisation bzw. Major- oder Minoramputationen bei annähernd stabilem Fontaine Stadium IV? Revaskularisation bei nicht unmittelbar drohendem Beinverlust?) oder chronischer Mesenterialinsuffizienz (Revaskularisation bei stabiler Angina abdominalis?). Zudem war auch das Vorgehen bei symptomfreiem Aortenaneurysma uneinheitlich: während nahezu alle Institutionen symptomfreie Aortenaneurysmen kleiner als $7 \mathrm{~cm}$ verschoben oder absetzten, wurden Aneurysmen mit Maximaldurchmesser größer $7 \mathrm{~cm}$ in $75 \%$ der Institutionen weiterhin behandelt.

Die Onlineumfrage war für die „Indikationen mit uneinheitlichem Management" möglicherweise zu wenig detailliert, d.h. eine weitere Ausformulierung der Fragen hätte vielleicht mehr Klarheit geschaffen. Jedenfalls sind die „Indikationen mit uneinheitlichem Management" wohl jene Themenbereiche, die künftig einer weiteren Festlegung bedürfen, und somit auch einer weiteren Forschungsaktivität (z. B. über den natürlichen Krankheitsverlauf), um herauszufinden, wie hier insgesamt besser selektioniert werden kann.

In der bisher publizierten Literatur finden sich u.a. Triage-Empfehlungen für die Gefäßchirurgie erstellt vom American College of Surgeons (ACS) [1]: diese empfehlen hinsichtlich der o.g. „Indikationen mit uneinheitlichem Management“ interessanterweise eine Intervention zu verschieben für Patienten mit symptomfreiem Aortenaneurysma kleiner als $6,5 \mathrm{~cm}$ („postpone“), aber auch für jene größer als $6,5 \mathrm{~cm}$ („postpone if possible"). Auch wird empfohlen, die Behandlung bei CLTI (Stadium III oder IV nach Fontaine) oder bei chronischer Mesenterialinsuffizienz falls vertretbar $\mathrm{zu}$ verschieben (jeweils „postpone if possible"). Hinsichtlich der Shuntchirurgie wird detailliert differenziert: Von einer Shuntneuanlage wird abgeraten 
(„consider postponing“), auch von einer Shuntrevision bzw. Shuntangiographie bei schlechter Funktion („postpone if possible“), jedoch wird eine Notfallintervention bei thrombosiertem Shunt bzw. Nichtfunktion, bei Steal oder bei Infekt empfohlen. Diese Empfehlungen des American College of Surgeons (ACS) stellen wohl in erster Linie einen Expertenkonsensus dar, jedenfalls wird keine Literatur als Beleg bzw. kein Evidenzlevel angegeben.

Eine in der Lockdown-Phase durchgeführte Onlineumfrage liefert naturgemäß keine Daten über die klinischen Ergebnisse der Patienten, sowohl jener, die behandelt wurden, als auch jener, deren Operation oder Intervention abgesetzt bzw. verschoben wurde. Diese Daten wären wichtig, v.a. für die abgesetzten bzw. verschobenen Patienten, werden aber schwer zu erheben sein (die Kohorte besteht auch aus den Patienten, die aufgrund der COVID-19-Pandemie niemals ärztlich untersucht wurden, oder jenen mit Komplikationen, die vielleicht auch ohne die COVID-19-Pandemie aufgetreten wären) und werden - falls überhaupt - wohl erst zu einem deutlich späteren Zeitpunkt (in 6 bis 12 Monaten?) der „Aufarbeitung“ der verschobenen Fälle erfasst werden können.

\section{》) Die Onlineumfrage liefert keine Daten über die klinischen Ergebnisse der Patienten}

Nicht zuletzt muss auch auf die - unabhängig von klassischen kardiovaskulären Ergebnisparametern - potenzielle zusätzliche psychologische Belastung der Patienten hingewiesen werden, die durch OP-Absagen und OP-Verschiebungen eingetreten ist. Die unklar befristete „Wartezeit", potenziell mögliche Komplikationen währenddessen und die Auswirkung der Verunsicherung auch auf die Lebensqualität der Patienten sind sicherlich nicht zu unterschätzen. Zusätzlich ungünstig könnte sich ausgewirkt haben, dass in der Wartezeit auch die Arzt-Patienten-Kommunikation nicht in üblicher Art und Weise erfolgen konnte, da die Patienten seitens der Gesundheitsbehörden aufgefordert wurden, „sich von Krankenhäusern nach Möglichkeit fern zu halten“.

\section{Ausblick}

Aufgrund der COVID-19-Pandemie erfolgte OP-Verschiebungen und Veränderungen im Alltag der Patienten (Verhaltensänderungen betreffend Lifestyle inkl. körperliche Aktivitäten und Psyche, zu erwartende Auswirkungen auf Risikofaktoren wie Rauchen, Alkoholkonsum und Diät) sollten hinsichtlich ihrer gesundheitlichen Auswirkungen nicht unterschätzt werden. $\mathrm{Zu}$ erwarten ist, dass diese Auswirkungen demografische und geografische Unterschiede zeigen, dass sich langfristig wirksame positive und negative gesundheitliche Konsequenzen zeigen und dass diese abhängig von den angeordneten Maßnahmen und deren zeitlicher Befristung sind. Alle diese Aspekte erfordern eine auf die Problemstellung angepasste und somit neuartige kritische wissenschaftliche Analyse inklusive der Einbeziehung neuer Konzepte im Alltag (z.B. visuelle und supportive Telemedizin), insbesondere auch in der Gefäßmedizin. Es gilt also wohl längerfristig das auf die COVID-19-Pandemie bezogene Zitat „we are only at the beginning of this journey together" [6].

\section{Fazit für die Praxis}

- Die COVID-19-Pandemie traf - sicherlich auch in Österreich - ein auf eine derartige Krise nur eingeschränkt vorbereitetes Gesundheitssystem.

- Entscheidungsträger, auch im Fachgebiet Gefäßchirurgie, waren gefordert, die Kapazitäten für Akutfälle und dringliche Fälle aufrecht zu erhalten, mussten aber Verschiebungen elektiver Operationen (mit primär unklarem Zeitfenster) anordnen.

- Für einzelne Indikationen (z. B. symptomfreies Aortenaneurysma größer als $7 \mathrm{~cm}$, chronische Ulzera 0 . chronische Mesenterialischämie, Shuntchirurgie) war das Vorgehen während der COVID-19-Pandemie in den befragten österreichischen Institutionen uneinheitlich.
- Diese Bereiche sollten hinsichtlich der klinischen Ergebnisse nach OPVerschiebungen, der Möglichkeiten zur detaillierteren Selektion und der Strategien zur Komplikationsvermeidung weiter analysiert und beforscht werden.

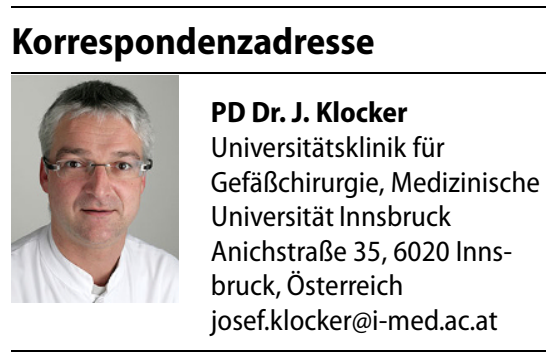

Mitglieder des Vorstandes der Österreichischen Gesellschaft für Gefäßchirurgie (ÖGG). PD Dr. J. Klocker, M. Kluckner, W. Hofmann, A. Assadian.

Danksagung. Die Autoren und die Mitglieder des Vorstandes der Österreichischen Gesellschaft für Gefäßchirurgie (ÖGG) bedanken sich bei den gefäßchirurgischen Abteilungen, die den Onlinefragebogen beantwortet haben.

Funding. Open access funding provided by University of Innsbruck and Medical University of Innsbruck.

\section{Einhaltung ethischer Richtlinien}

Interessenkonflikt. J. Klocker, A. Frech, A. Gratl, M. Thaler, I. Khosravi, M. Liebensteiner, M. Kluckner, W. Hofmann und A. Assadian geben an, dass kein Interessenkonflikt besteht.

Für diesen Beitrag wurden von den Autoren keine Studien an Menschen oder Tieren durchgeführt. Für die aufgeführten Studien gelten die jeweils dort angegebenen ethischen Richtlinien.

Open Access. Dieser Artikel wird unter der Creative Commons Namensnennung 4.0 International Lizenz veröffentlicht, welche die Nutzung, Vervielfältigung, Bearbeitung, Verbreitung und Wiedergabe in jeglichem Medium und Format erlaubt, sofern Sie den/die ursprünglichen Autor(en) und die Quelle ordnungsgemäß nennen, einen Link zur Creative Commons Lizenz beifügen und angeben, ob Änderungen vorgenommen wurden.

Die in diesem Artikel enthaltenen Bilder und sonstiges Drittmaterial unterliegen ebenfalls der genannten Creative Commons Lizenz, sofern sich aus der Abbildungslegende nichts anderes ergibt. Sofern das betreffende Material nicht unter der genannten Creative Commons Lizenz steht und die betreffende Handlung nicht nach gesetzlichen Vorschriften erlaubt ist, ist für die oben aufgeführten Weiterverwendungen des Materials die Einwilligung des jeweiligen Rechteinhabers einzuholen. 
Weitere Details zur Lizenz entnehmen Sie bitte der Lizenzinformation auf http://creativecommons.org/ licenses/by/4.0/deed.de.

\section{Literatur}

1. American College of Surgeons (2020) COVID19: elective case triage guidelines for surgical care. https://www.facs.org/covid-19/clinical/ guidelines/elective-case. Zugegriffen: 24. März 2020

2. Clerkin KJ, Fried JA, Raikhelkar J, Sayer G, Griffin JM, Masoumi A et al (2020) Coronavirus disease 2019 (COVID-19) and cardiovascular disease. Circulation 141(20):1648-1655

3. COVID Collaborative (2020) Elective surgery cancellations due to the COVID-19 pandemic: global predictive modelling to inform surgical recovery plans. Br J Surg. https://doi.org/10.1002/ bjs.11746

4. Verity R, Okell LC, Dorigatti I, Winskill P, Whittaker C, Imai $\mathrm{N}$ et al (2020) Estimates of the severity of coronavirus disease 2019: a model-based analysis. Lancet Infect Dis. https://doi.org/10.1016/S14733099(20)30243-7

5. Liebensteiner MC, Khosravi I, Hirschmann MT, Heuberer PR, Board of the AGA (2020) Massive Cutback in orthopaedic healthcare services due to the COVID-19 pandemic. Knee Surg Sports Traumatol Arthrosc 28(6):1705-1711

6. Liu PP, Blet A, Smyth D, Li H (2020) The science underlying COVID-19. Implications for the cardiovascular system. Circulation 142:68-78

7. Thaler M, Khosravi I, Hirschmann MT, Kort NP, Zagra L, Epinette JA, Liebensteiner MC (2020) Disruption of joint arthroplasty services in Europe during the COVID-19 pandemic: an online survey within the European Hip Society (EHS) and the European Knee Associates (EKA). Knee Surg Sports Traumatol Arthrosc 28(6):1712-1719

\section{Schlaganfallgefahr durch verengte Halsschlagader Kontrolluntersuchungen und OP-Termine unbedingt wahrnehmen}

In Deutschland leben etwa eine Million Menschen mit einer Halsschlagader, die zu mehr als $\mathbf{5 0} \%$ durch Kalkablagerungen verengt ist - lösen sich aus den Plaques Stückchen heraus, droht ein Schlaganfall im Gehirn. Viele dieser Patienten haben aus Angst vor einer Corona-Infektion notwendige Kontrolluntersuchungen oder anstehende Operationen vermieden. Die Deutsche Gesellschaft für Gefäßchirurgie und Gefäßmedizin e.V. (DGG) rät, diese Termine dennoch unbedingt wahrzunehmen.

„Wir befinden uns fast wieder im Normalbetrieb", betont DGG-Experte PD Dr. med. Farzin Adili. „Die Angst vor einer CoronaInfektion im Krankenhaus ist weitgehend unbegründet", fügt der Chefarzt der Klinik für Gefäßmedizin, Gefäß- und Endovascularchirurgie am Klinikum Darmstadt hinzu. Patienten sollten vor einem Klinikaufenthalt einen Corona-Abstrich vornehmen lassen. „Andernfalls müssen wir den Abstrich in der Klinik machen”, so Adili. „Aber auf keinen Fall sollten Kontrolluntersuchungen und Operationen von Gefäßerkrankungen auf die lange Bank geschoben werden."

\section{$15 \%$ aller durchblutungsbedingten Schlaganfälle}

In Deutschland erleiden jedes Jahr bis zu 30.000 Menschen einen Schlaganfall, der von Ablagerungen an der Gefäßwand einer verengten Halsschlagader ausgeht. Das entspricht $15 \%$ aller Schlaganfälle, die durch Durchblutungsstörungen hervorgerufen werden. Die Carotisstenose fällt häufig lange Zeit nicht auf, weil sie zunächst keine Beschwerden verursacht und die Halsschlagader der Gegenseite eine etwaige Minderdurchblutung ausgleichen kann.

Eine Ultraschall-Untersuchung kann die Ablagerungen aber sichtbar machen. Die DGG empfiehlt deshalb ein jährliches UltraschallScreening der Carotis ab dem Lebensalter von 65 Jahren, wenn Risikofaktoren vorliegen. Zu diesen Risiken zählen Herzerkrankungen, Diabetes mellitus, die periphere arterielle Verschlusskrankheit (pAVK), Nikotinkonsum oder Bluthochdruck.

Neue Leitlinie: Ab 50-\%iger Verengung und Durchblutungsstörungen kommt ein Eingriff in Frage

Bei Menschen, die eine Halsschlagaderverengung aufweisen, jedoch keine Symptome zeigen, kann meist zunächst abgewartet, medikamentös behandelt und regelmäßig kontrolliert werden. Problematisch wird es jedoch, wenn sich an der Oberfläche der Ablagerungen Blutgerinnsel bilden, die sich von Zeit zu Zeit ablösen und immer wieder Blutgefäße im Gehirn verstopfen. Es kommt dann zu vorübergehenden oder auch dauerhaften Durchblutungsstörungen des Gehirns mit neurologischen Ausfallerscheinungen - einem Schlaganfall. Die Ausfälle zeigen sich in Form von Lähmungserscheinungen oder Kribbeln an Händen, Armen oder Beinen einer Körperhälfte, seitenbetontem Schwächegefühl oder dem Einknicken eines Beines. Weitere Alarmzeichen sind Sprach- oder einseitige Sehstörungen. Bei solchen Symptomen und einer Verengung der Halsschlagader auf beiden Seiten von mehr als $50 \%$ sollte eine Operation durchgeführt werden, um die Ablagerungen zu entfernen. So empfiehlt es auch die neue Experten-Leitlinie zur Carotisstenose aus dem Februar dieses Jahres.

Für die Operation an der Halsschlagader gilt: Sie gehört zu den wissenschaftlich bestuntersuchten Eingriffen und ist sicher, sofern der Gefäßchirurg über entsprechende Expertise verfügt. Es ist gesetzlich vorgeschrieben, die Ergebnisse der Carotis-Operationen zu melden. Sie werden in jährlichen Qualitätsberichten angegeben. Patienten können sich bei ihrem Gefäßchirurgen danach erkundigen.

Literatur:S3 Leitlinie zur Diagnostik, Therapie und Nachsorge der extracraniellen Carotisstenose:

https://www.awmf.org/leitlinien/detail/Il/004028.html

Deutsche Gesellschaft für Gefäßchirurgie und Gefäßmedizin e.V. (DGG), www.gefaesschirurgie.de 